\title{
Elementos que influenciam o acesso à atenção primária na perspectiva dos profissionais e dos usuários de uma rede de serviços de saúde do Recife
}

\author{
I 1 Sayonara Arruda Vieira Lima, ${ }^{2}$ Maria Rejane Ferreira da Silva, \\ ${ }^{3}$ Eduardo Maia Freese de Carvalho, ${ }^{4}$ Eduarda Ângela Pessoa Cesse, \\ ${ }^{5}$ Ederline Suelly Vanini de Brito, ${ }^{6}$ João Paulo Reis Braga I
}

Resumo: Apesar de todo o arcabouço institucional e do aparato jurídico-legal do SUS criado para melhorar o acesso da população aos serviços de saúde, ainda existem muitas barreiras que interferem na concretização do acesso universal à saúde no Brasil. Objetivamos analisar os elementos que influenciam o acesso aos serviços de atenção primária no município de Recife na perspectiva de profissionais e usuários. Trata-se de um estudo de caso realizado através de entrevistas semiestruturadas com46 informantes. A análise de conteúdo foi o método escolhido para trabalhar os dados. As principais barreiras que emergiram da pesquisa foram o subfinanciamento dos serviços, a cobertura ainda insuficiente da Estratégia de Saúde da Família (ESF) e a oferta insuficiente de profissionais. Entre os elementos facilitadores, os mais relevantes foram a própria existência da ESF, a comunicação informal e o trabalho do agente comunitário de saúde. Conclui-se que, mesmo com a implantação da ESF e dos benefícios gerados, ainda há a necessidade de a estratégia ser uma prioridade da gestão, com maior investimento na estrutura e na organização dos serviços ofertados para que os usuários tenham acesso universal e equânime à rede de saúde de Recife.

\author{
1 Centro de Pesquisas Aggeu \\ Magalhães. Fiocruz-NESC. \\ Recife-PE, Brasil. Endereço \\ eletrônico: sayonaraarruda@ \\ gmail.com \\ ${ }^{2}$ Centro de Pesquisas Aggeu \\ Magalhães. Fiocruz-NESC. \\ Recife-PE, Brasil. Endereço \\ eletrônico: rejane.ferreira@ \\ upe.br \\ ${ }^{3}$ Centro de Pesquisas Aggeu \\ Magalhães. Fiocruz-NESC. \\ Recife-PE, Brasil. Endereço \\ eletrônico: freese@cpqam. \\ fiocruz.br \\ ${ }^{4}$ Centro de Pesquisas Aggeu \\ Magalhães. Fiocruz-NESC. \\ Recife-PE, Brasil. Endereço \\ eletrônico: educesse@uol. \\ com.br \\ ${ }^{5}$ Universidade de Pernambuco. \\ Recife-PE,Brasil. Endereço \\ eletrônico: ederline_vanini@ \\ yahoo.com \\ ${ }^{6}$ Universidade de Pernambuco. \\ Recife-PE,Brasil. Endereço \\ eletrônico: jpreisbraga@ \\ hotmail.com
}


Durante a década de 90, foram realizadas reformas nos sistemas de saúde em diversos países da América Latina com a proposta da implantação das Redes Integradas dos Serviços de Saúde (RISS). Essas redes são definidas como um conjunto de organizaçooses que oferece continuamente, de forma direta ou através da compra, serviços de saúde a uma população estabelecida, e que se responsabiliza pelos custos e resultado em saúde de sua população (SHORTELL; GILLIES; ANDERSON, 1994).

A implantação dessas redes tem como objetivos intermediários o acesso à atenção à saúde, a continuidade assistencial e a coordenação assistencial. Seus objetivos finais são a equidade de acesso e a eficiência dos serviços de saúde. Tais resultados são importantes para analisar o desempenho das redes (VÁZQUEZ et al., 2009). A integração dos serviços de saúde em RISS como forma organizar a prestação de atenção deve ser um meio para melhorar a eficiência e o acesso aos serviços, e não um fim em si mesma, principalmente quando não se conhecem exatamente seus potenciais benefícios e riscos (VÁZQUEZ; VARGAS, 2006).

De acordo com a Organização Pan-americana da Saúde (2010), as redes integradas de serviços de saúde devem apresentar alguns atributos essenciais para um adequado funcionamento. Dentro dos âmbitos de abordagem há os atributos relacionados ao modelo assistencial.

O modelo assistencial deve ter um território e uma população definidos e um amplo conhecimento das necessidades e demandas de saúde, sendo o modelo quem determina a oferta dos serviços de saúde. Deve existir uma extensa rede que oferte serviços relacionados à promoção da saúde até a reabilitação e os cuidados paliativos e que integre programas de doenças e agravos específicos, riscos e populaçõos específicas, os serviços de saúde privados e os serviços de saúde pública. Além disso, o primeiro nível de atenção multidisciplinar deve cobrir toda a população e servir como porta de entrada do sistema, que integra e coordena a atenção à saúde, além de responder à maior parte das necessidades de saúde da população. O modelo deve, finalmente, apresentar um mecanismo de coordenação da assistência ao longo de toda a rede dos serviços de saúde, e a atenção deve ser centrada no indivíduo, na família e na comunidade (ORGANIZAÇÃO PAN-AMERICANA DE SAÚDE, 2010). 
No Brasil, em 2011, mais de vinte anos após a criação do Sistema Único de Saúde (SUS), em face de lacunas legais quanto à organização do sistema, ao planejamento da saúde, à assistência à saúde e à articulação interfederativa, ainda existe a necessidade de regulamentação de dispositivos da Lei no 8.080, por meio do Decreto no 7.508 . Esse documento estabelece para a organização do SUS, as Regiōes de Saúde para provisão dos serviços, cumprindo a determinação constitucional de que o SUS é composto por uma rede regionalizada e hierarquizada (BRASIL, 2011).

De acordo com a Portaria no 4.279, para assegurar a resolutividade na rede de atenção, um dos fundamentos que precisa ser considerado é o acesso, que pode ser analisado através (I) da disponibilidade, que diz respeito à obtenção da atenção necessária ao usuário e sua família; (II) da comodidade, relacionada ao tempo de espera para o atendimento, à conveniência de horários, à forma de agendamento, à facilidade de contato com os profissionais, ao conforto dos ambientes para atendimento, entre outros; e (III) da aceitabilidade, relacionada à satisfação dos usuários quanto à localização e à estrutura física do serviço, ao tipo de atendimento prestado, e relacionada, também, à aceitação dos usuários quanto aos profissionais responsáveis pelo atendimento (BRASIL, 2010).

Um dos modelos teóricos existentes para o estudo sobre o acesso aos serviços de saúde foi apresentado por Aday e Andersen (1974). Esses autores apresentaram a proposta do modelo comportamental sobre o uso dos serviços de saúde. Partindo das políticas de saúde, traçava-se uma rede de variáveis que incluíam as características do sistema de saúde (recursos e organização) e da população em risco (predisposição, capacidades e as necessidades percebidas e avaliadas pelos profissionais de saúde), a utilização dos serviços e a satisfação dos consumidores.

Quase três décadas depois de implantação e da implementação do SUS, ainda se observam diversos problemas de acesso dos brasileiros aos serviços de saúde. Para uma adequada compreensão desses problemas, são necessárias mais evidências a respeito do impacto das mudanças em relação ao acesso da população adscrita, bem como evidências que estabeleçam se essa adscrição se traduz em maior possibilidade de acesso aos serviços.

Em busca de evidências após as mudanças realizadas, este estudo analisa os elementos que influenciam o acesso aos serviços de saúde de Recife, Pernambuco, com o objetivo de identificar as barreiras e os elementos facilitadores do acesso 
aos serviços de saúde da atenção primária na visão dos profissionais de saúde e dos usuários.

Destaca-se que o presente artigo é um recorte do projeto de pesquisa intitulado "Impacto na equidade de acesso e eficiência de Redes Integradas de Serviços de Saúde na Colômbia e Brasil - Equity-LA", que tem como um dos objetivos específicos estabelecer e analisar as opiniōes dos atores-chave sobre o acesso às redes integradas de serviços de saúde (VÁZQUEZ et al., 2013).

\section{Procedimentos metodológicos}

Trata-se de um estudo de caso, com abordagem qualitativa, que segue o estabelecido na metodologia da pesquisa Equity-LA (VÁZQUEZ et al., 2013). O estudo foi realizado no município de Recife, selecionado como caso do projeto por apresentar as seguintes características: é um município com a gestão plena do sistema de saúde; apresenta uma rede que proporciona continuidade na assistência à população adstrita (atenção primária e especializada); possui uma distinta proporção de titularidade da rede pública e conveniada (privada) e distinta proporção da população coberta por equipes de saúde da família.

Dentro do município foi escolhida a rede de saúde do Distrito Sanitário III, especificamente na microrregião 3.3, localizada no noroeste de Recife, com 11.502 habitantes (7\% da cidade) (VÁZQUEZ et al., 2013), e formada por oito bairros (CAVALCANTI; LYRA; AVELINO, 2008). A rede selecionada oferece serviços de atenção primária, média e alta complexidade.

Foram selecionados como participantes da pesquisa atores-chave que poderiam ter uma opinião sobre o acesso ao longo da assistência contínua. Foram realizadas quarenta e seis entrevistas. As categorias, a quantidade de entrevistados por categoria e os critérios para a seleção desses profissionais foram: nove gerentes de serviços de atenção primária e atenção especializada (GE) que ocupavam uma posição de direção na prestação de saúde; seis profissionais administrativos (PA), que trabalhavam em serviços de apoio dos prestadores relacionados à coordenação do paciente com outros níveis assistenciais (admissões, atenção ao usuário, serviço de referência e contrarreferência de pacientes, trabalho social, auditoria médica, etc.), tendo trabalhado na instituição por mais de um ano; dezessete profissionais de saúde de cada nível assistencial (Atenção Primária - PSI e Atenção Especializada - PSII), com atividade de pelo menos seis meses no centro; quatorze usuários (com 
e sem cobertura por equipes de saúde da família). Para os usuários cobertos, foram

aplicados como critério ter utilizado o primeiro nível assistencial e tentado utilizar ou ter utilizado a atenção especializada nos seis meses anteriores à entrevista; para não cobertos, ter tentado utilizar algum serviço de saúde nos seis meses anteriores à entrevista. E, em ambos os casos, ser maior de 18 anos.

Os profissionais foram escolhidos nas unidades prestadoras de serviços de saúde, selecionadas a partir dos seguintes critérios: prestadores de serviços existentes dentro e fora da área de estudo (rede virtual), de distintos níveis assistenciais (I, II e III nível) e que atendessem aos pacientes procedentes da área de estudo.

As entrevistas foram realizadas individualmente, no período de outubro de 2009 a junho de 2011. Os dados foram coletados nas unidades de saúde em que os profissionais trabalhavam, nos domicílios dos usuários ou em um espaço escolhido pelo próprio entrevistado, por meio de entrevistas semiestruturadas guiadas por um roteiro temático pré-estabelecido, específico para cada grupo de informantes, que incluía perguntas relacionadas ao acesso. Todas as entrevistas foram gravadas em meio digital e transcritas. Foram escolhidas duas categorias teóricas para serem trabalhadas neste artigo: barreiras do acesso e os elementos facilitadores no uso dos serviços de saúde. O processo de análise iniciou-se com ajuda do software Atlas TI 5.0, e os dados foram analisados através da técnica de análise de conteúdo de Bardin (2011).

A pesquisa foi aprovada pelo Comitê Nacional de Ética em Pesquisa (CAAE 35050000 250-08) e autorizada pela secretaria de saúde de Recife e pela Secretaria Estadual de Saúde.

\section{Resultados e discussão}

\section{Barreiras ao acesso relacionadas com a gestão da rede}

De acordo com os profissionais da atenção primária, a influência política é uma barreira que altera a prioridade do uso dos recursos financeiros destinados à saúde. Há uma interferência na tomada de decisão para escolha de quais programas irão permanecer, e muitas vezes eles mudam de acordo com a gestão que está no poder, e não por critérios epidemiológicos. Como consequência, muitos programas de saúde não têm a continuidade das suas atividades, o que dificulta a atuação dos profissionais e o seguimento do tratamento dos pacientes. 

treinada na outra gestão pra tabagismo. Ninguém mais falou em tabagismo. Cadê meu dipobiona, minha... minha nicotina de mascar e cadê meu adesivo? Cadê o programa? (PSI 5).

Ainda conforme a opiniāo dos profissionais da atenção primária, a influência política também acontece no cotidiano das atividades dos serviços. As decisões tomadas pelos profissionais de saúde, muitas vezes, favorecem a determinados usuários em detrimento de outros, assim como o conselho de saúde, que, muitas vezes, aprova medidas que geram benefícios pessoais, com a intenção de conquistar um ganho de cunho político, e não para proporcionar melhorias de acordo com a necessidade do sistema de saúde.

Aí tem as questôes políticas também, alguns agentes de saúde favorecem alguns e outros favorecem a outros, tudo isso está junto. Eu acho que tudo isso também influencia. [...] A questão política também em muitos lugares complica. Tem conselho de saúde que interfere muito no processo, até numa forma positiva. Não estou dizendo que é... Às vezes, é positivo, mas, às vezes, é tão emaranhado de interesses ali, tão grande, que não é propriamente o interesse da saúde, das pessoas que tão naquela área (PSI 4).

Um profissional da atenção primária também menciona a questão da influência política como barreira ao acesso no trabalho realizado pelos líderes nas comunidades. Estes atuam para obter vantagens para os próprios interesses, cobrando melhorias em períodos nos quais consigam se beneficiar politicamente, em vez de realmente realizar o controle social e exigir as modificações necessárias para qualificar os atendimentos nas unidades de saúde.

Essa questão da liderança, de organizar mesmo politicamente, ainda também está muito politiqueira. Está muito arraigada a partidos. A gente não vê muita liderança da comunidade envolvida querendo melhorar, caminhando junto com o PSF [Programa Saúde da Família]. A gente vê muito essa questão ainda da política pequena. [...] Então assim, ele vê ainda essa coisa da organização mesmo, como: "ah não, quando chegar a época de política, eu vou trabalhar na campanha”. (PSI 2).

A outra barreira relacionada à gestão que emergiu principalmente dos discursos dos gerentes foi a questão do financiamento dos serviços. Os entrevistados citaram a falta de incentivo, que impede a continuidade das açóes, como resultado da insuficiente contrapartida de recursos repassados pelo Governo Federal para o fortalecimento da ESF. A verba destinada à atenção primária é escassa para as despesas destinadas à manutenção da cobertura do programa e a investimentos 
em ampliação e melhoria da resolubilidade das equipes de saúde da família.

Como consequência, tem-se frustração e perda de credibilidade da população que depende dos serviços.

Falta a fixação da contrapartida federal mesmo, pra colaborar nessa fixação dessas equipes e a implementação dela. Muitas vezes você tem abrangência de 70\% e 60\% do PSF, mas qual a resolutividade deles? Você precisa estar junto pra resolver, não só para estar junto. (GE 6).

Eu tive esse serviço (ortodontia), mas o serviço parou por falta de recurso. Aí fiquei triste, porque o serviço tão bem feito, fui tão bem tratado, eu pensei que ia continuar, mas parou por falta de recurso [...] (USD 5).

Essa constatação é colocada por Silva (2011) como um dos desafios para as redes regionalizadas e integradas. Apesar de a cobertura assistencial no SUS ter aumentado nas duas últimas décadas, especialmente na área ambulatorial, a oferta de recursos ainda é insuficiente para atender às necessidades da população. A expansão se deu praticamente pelos municípios, induzidos pelo governo federal por transferências de recursos financeiros através de incentivos para implantar serviços e programas, e existem indícios de que há um esgotamento nessa forma de expansão.

Além disso, para Gadelha et al. (2011), a distribuição regional dos investimentos, em geral, não segue estratégias de longo prazo. Sua efetividade também é limitada pela fragmentação e pelo porte de recursos: os fluxos financeiros do SUS tendem a se direcionar para os locais onde já há infraestrutura instalada e mão de obra qualificada.

\section{Barreiras ao acesso relacionadas com \\ a estrutura e a organização dos serviços}

Do discurso dos entrevistados, emergiram elementos de natureza estrutural e organizacional dos serviços que dificultam o acesso à atenção primária à saúde (APS).

Constituíram-se como elementos estruturais: a oferta dos serviços disponíveis; a disponibilidade de recursos humanos; a disponibilidade de insumos e a acessibilidade geográfica. No que se refere aos aspectos organizacionais, foram identificados como elementos que limitam o acesso às visitas domiciliares e o tempo de espera. 


\section{Elementos estruturais que limitam o acesso}

$\mathrm{Na}$ opinião da maior parte das categorias profissionais, há uma oferta limitada da ESF, estando muitas áreas do município de Recife descobertas pelas equipes de saúde da família. Até mesmo em uma equipe pode não haver cobertura de toda a área adstrita por falta de agentes comunitários de saúde (ACS), gerando uma desigualdade de acesso dos usuários dentro do território.

Já foi dito inclusive pelo próprio pessoal da prefeitura do Recife que os PSF infelizmente não conseguem cobrir toda área. Então nem todos os distritos são totalmente cobertos. A gente que pega aqui a área de Casa Amarela, pega o distrito dois e três, e nem todos são cobertos por equipe de PSF. (PA 5)

Apesar de o município de Recife, entre 2000 a 2008, ter tido uma enorme expansão da rede de saúde em todos os níveis de atenção, inclusive com a ampliação da cobertura pelas unidades de saúde da família (USF) (CÔELHO; COUTO, 2009), de acordo com os dados do Departamento de Atenção Básica (DAB/MS) sobre a cobertura no município, há um registro estimado de 53,02\% de adscrição da população pelas equipes de saúde da família (BRASIL, 2013); ou seja, quase metade da população está descoberta.

Todos os grupos destacaram que há uma oferta insuficiente de médicos e outros profissionais na atenção primária para a demanda existente. Esse problema gera um aumento no tempo para a população conseguir atendimento, e muitas vezes a qualidade da consulta não responde às necessidades do paciente. Além disso, há uma sobrecarga de trabalho para os outros profissionais da equipe e a busca por parte dos usuários pelos serviços de alta complexidade.

A população para a quantidade de profissional é pouca. Por exemplo, clínico, que tem lá em Nova Descoberta, deveria ter mais gente pra atender. [...] Dentista também é pouco, daquela população de lá. Então, isso aí é uma dificuldade ao acesso, tem gente que passa muito tempo para ser atendido. Mas não é porque não tem. É porque é insuficiente. (GE 3).

Tal resultado também é encontrado por Vanderlei e Navarette (2013), no município de Recife. Os autores enfatizam a falta de profissionais como uma dificuldade de acesso à saúde. Para corroborar com a informação anterior, dados mostram que o número de médicos generalistas por 1.000 habitantes variou de 0,3 a 2,1 nos países europeus em 2005. Já no Brasil, os médicos do PSF, que em tese seriam os generalistas do SUS, correspondem a apenas 0,1 por 1.000 hab. Somando a eles os que atuam nas especialidades básicas - ginecologia/obstetrícia, clínica geral e pediatria - a taxa é de 0,63 (CONSELHO NACIONAL..., 2008). 
Também foi considerada como barreira, principalmente pelos usuários, a falta

de insumos para o atendimento e tratamento. De acordo com os informantes, há uma escassez de medicamentos que deveriam ser disponibilizados gratuitamente para a população, bem como de materiais para realizar determinados procedimentos nas unidades. Assim, o tratamento é interrompido ou torna-se impossível o seguimento da terapia recomendada, pois, muitas vezes, os usuários não têm recursos financeiros para comprá-los.

Quando chega um paciente aqui, a gente não tem resolutividade. Não tem uma medicação para que possa fazer baixar rápido a pressão do paciente. Não tem uma medicação injetável, que talvez não é nem para ter, mas talvez tivesse, talvez fosse revista, seria até para fazer uma sutura na unidade básica. (PSI 3)

Esse aspecto também apareceu como resultado em pesquisas realizadas por Vanderlei e Navarette (2013) e Moimaz et al. (2010). Estes últimos autores alertam que a queixa da falta de medicamentos deve ser analisada com cautela, porque se deve considerar a existência de uma limitação de recursos financeiros para a compra de remédios nos municípios, o que gera uma cobertura incompleta das necessidades dos usuários.

Com relação à acessibilidade geográfica, principalmente os profissionais da atenção primária e os usuários não cobertos relatam a dificuldade no acesso geográfico aos serviços de saúde devido à distribuição inadequada das USF dentro do território, havendo grande distância entre a residência dos usuários e os serviços de atendimento, bem como a presença de morros, escadarias e córregos no trajeto.

Ela está funcionando como um posto grande como esse, com várias equipes dentro e os usuários se deslocando das suas comunidades, das barreiras, escadarias, dos locais de difícil acesso, estão vindo para esta unidade. [...] A questão da dificuldade de acesso mesmo, da localidade, de distância. (PSI 2).

A localização inadequada das USF foi citada como barreira de acesso em Coimbra et al. (2010). Muitas vezes as unidades se encontram distantes de muitas das microáreas adscritas, além de apresentarem muitas dificuldades físicas do território como escadarias, encostas e ladeiras.

No relato dos usuários, identificou-se que a divisão do território para definir a área de cobertura das USF é um mecanismo organizacional que, algumas vezes, dificulta o uso do serviço mais próximo a suas residências.

Diz que o acesso para a gente é P.B., sabe onde fica o posto P.B.? É um posto que fica na BR, entrando na BR, lá pra dentro mesmo, fica bem distante. Minha filha sai com 
Para Silva Júnior et al. (2010), essa realidade ocorre porque o processo de adscrição dos usuários, ao não considerar a dinâmica do território, suas características e seu perfil de saúde-doença, representa uma atividade meramente burocrática: as áreas das equipes e suas microáreas são apenas espaços delimitados para atender às exigências formais de funcionamento dos programas. Observa que existe uma lógica estritamente normativa, dado que a definição das áreas pode ser baseada exclusivamente no número máximo de pessoas por ACS.

\section{Elementos organizacionais que limitam o acesso}

Apesar de a ESF ser pensada para aumentar as ações de prevenção e promoção, ainda permanece a lógica de um modelo curativista, com prioridade para as atividades realizadas dentro do consultório. Os usuários, principalmente, consideraram reduzido o número de visitas domiciliares realizadas tanto pelos profissionais de nível superior quanto pelos ACS. Uma das justificativas é a alta demanda de consultas dentro da unidade, não sobrando tempo para as domiciliares, restringindo o acesso aos usuários que não podem se deslocar.

A população diz que precisa só de consulta. Eles querem o tempo todo ter uma con-
sulta individual. E o médico esta ali, naqueles entraves dele, o tempo todo no consul-
tório. As visitas que são mais importantes nem sempre se tem tempo para serem feitas.
Porque é nas visitas que você conhece a família e todas as dificuldades que tem ali. E
as visitas são poucas. (PSI 2).

Tal dado também foi encontrado em uma pesquisa realizada por Drulla et al.(2009), cujos informantes alegaram falta de tempo para a realização das visitas domiciliares por causa da sobrecarga de trabalho, sua reduzida duração devido a outras tarefas e a necessidade de se cumprir uma quantidade determinada para as visitas mensais.

Outra importante barreira mencionada com intensidade pelos usuários foi a respeito do prolongado tempo de espera para conseguir marcar as consultas. Os usuários precisam chegar muito cedo à unidade, pernoitar ou madrugar, e esperar horas para conseguir pegar uma ficha.

Ai a gente chega lá e fica lá esperando até 7 h da manhã para abrir o portão pra todo mundo entrar, pra poder marcar, começar a marcar. Tem que chegar de quatro horas, tem gente que dorme lá. (USC 1). 
Corroboram com os resultados encontrados os dados das pesquisas de Schwartz et al. (2010) e Coimbra et al. (2010). Neles, os usuários também apontam a dificuldade para a marcação de consulta e a necessidade de chegarem muito cedo para tentar conseguir uma ficha.

Os usuários entrevistados neste estudo também expressaram que outra dificuldade é o horário de atendimento insatisfatório durante o dia e a falta de atendimento no turno da noite e nos finais de semana. Além disso, há uma limitação de dias para a marcação da consulta: o usuário só tem a sua necessidade resolvida se estiver com o atendimento agendado para aquele dia.

Começa de 7 h e passa até 16 h só. Errado! [...] Depois de quatro horas não atende mais
ninguém. [...] Porque é o mais próximo da casa da gente, e numa hora que a gente está
mais necessitada está fechado. [...] Se esse posto fosse aberto por essa hora e a gente
chegasse qualquer hora e fosse atendida era bom. (USC 1).

Tal achado também é encontrado em uma pesquisa realizada por Escorel et al. (2007) em 10 municípios brasileiros com mais de 100.000 habitantes. Nela, há relatos de que as unidades só funcionavam das 8 às 17 horas, e que essa condição dificultava o acesso da população trabalhadora.

Um problema que reduz ainda mais a quantidade de atendimentos por dia, emergido dos discursos de todos os grupos, foi o descumprimento das oito horas de trabalho determinados para USF. A maioria dos entrevistados considerou que o desrespeito do médico ao horário de trabalho é mais expressivo que o dos demais profissionais, que geralmente têm outros empregos.

Porque tinha um caso de um PSF que o médico chegava, ele trabalhava na rede privada, chegava às dez (horas)... e onze e pouca ele já saía, e a enfermeira achava que... tava muito bom, porque ela passou muito tempo que nem médico tinha pra atender as pessoas, e apareceu esse que atendia nesse tempinho e ela ficou contemplada! E a população? Fazia um arremedo de assistência, né? (GE 4).

Apesar de ser estabelecido que a jornada de trabalho dos profissionais da equipe de saúde da família é de 40 horas semanais em Recife, nem sempre esse horário é cumprido de acordo com a Portaria no 648GM (BRASIL, 2006). Podese constatar em Pinto, Menezes e Villa (2010) que essa realidade se repete em outros locais, nos quais a jornada de trabalho é variável. Tanto os médicos como os enfermeiros cumprem uma carga horária de 6 horas diárias. Já os odontólogos cumprem 5 horas diárias. Os profissionais de nível médio, ACS e auxiliares de enfermagem, por sua vez, cumprem 8 horas diárias de trabalho, enquanto os auxiliares de saúde bucal (ASB) trabalham 6 horas. 


\section{Barreiras ao acesso relacionadas às características da população}

Os profissionais da atenção primária, principalmente, consideraram que, por causa da precária condição socioeconômica da população assistida, há uma dificuldade de acesso. Quando a unidade está distante de casa ou não podem se deslocar a pé, muitos dos usuários não têm condições de pagar um transporte para se deslocar para os serviços, nem para comprar medicamentos. Isso os impede de seguir o tratamento recomendado, provocando a ausência de resolutividade do problema.

A comunidade de saúde é muito longe do local onde a gente atende. Eles vêm andando, porque eles também não têm dinheiro para estar pegando transporte. Tem até ônibus na porta deles, de lá pra cá, mas eles não têm dinheiro pra vir. (PSI 3)

Eu não sei se vou comprar o remédio, porque a gente já não está podendo, com o dinheiro que a gente ganha. É que nem essa bolsa família que nem eu disse, graças a Deus! Para comprar pão e comprar ovo e ainda comprar remédio caro. Como é que vai poder comprar remédio caro? [...] (USC 1).

Em pesquisa realizada por Silva Júnior et al. (2010), foi mostrado que a principal forma de deslocamento utilizada pela população foi o deslocamento a pé, fato constatado em todas as equipes. Essa preferência estava mais relacionada ao custo do deslocamento, já que se tratava de áreas carentes e da própria distância da residência até as USF, mais do que da disponibilidade de transporte coletivo. Em Azevedo e Costa (2010), usuários e profissionais relatam que a baixa condição socioeconômica limita o acesso aos medicamentos, quando não disponíveis na unidade, porque os usuários não podem comprá-los.

Outra barreira expressa com ênfase pelos gerentes e profissionais da atenção primária foi a falta de informação dos usuários sobre os serviços de atenção primária, que desconhecem qual o fluxo ou a unidade que devem procurar de acordo com suas necessidades. A desinformação do usuário é apontada como consequência da inexistência de divulgação adequada nos serviços primários e da falta de oportunidade ou interesse da população em assistir às palestras educativas ofertadas nas USF.

O pessoal que vem, às vezes, não tem conhecimento de que ali não faz uma sutura, de que ali não tem um neurologista. A gente precisa estar o tempo todo informando. Então, às vezes, o usuário não tem essa visão do que é o serviço de atenção básica, o que é o serviço de média complexidade e de alta complexidade. Não são todas as pessoas, mas a maioria dos usuários. (PSI 2). 
Diante da problemática, Schwartz et al. (2010) ressaltam o papel dos profissionais que atuam na equipe de saúde da família de informar, de forma clara e acessível, a respeito da hierarquização da rede de saúde, explicando as opções de procedimentos que o serviço tem a oferecer. Percebe-se que muitas das expectativas dos usuários quanto à USF estão além do rol de atividades a serem ofertadas na APS, e ainda não está claro para a população o processo de descentralização da rede de serviços.

Tais resultados são apresentados em um quadro-síntese (Quadro 1), abaixo, das barreiras ao acesso aos serviços de saúde no DS III do município de Recife, relacionados à gestão, estrutura, organização dos serviços e às características da população.

QUADRO 1. Barreiras ao acesso aos serviços de saúde relacionados com a gestão, com a estrutura, com a organização dos serviços e com as características da população. Microrregiāo 3.3, DS III. Recife-PE, 2011

\begin{tabular}{|l|l|l|}
\hline Categorias & Subcategorias & Subcategorias emergentes \\
\hline \multirow{5}{*}{ Gestão } & Política na gestão & $\begin{array}{l}\text { - Influência política na gestão das redes. } \\
\text { Citado por PSI; }\end{array}$ \\
\cline { 2 - 3 } $\begin{array}{l}\text { Estrutura } \\
\text { dos serviços } \\
\text { saúde }\end{array}$ & $\begin{array}{l}\text { Oferta dos serviços } \\
\text { disponíveis }\end{array}$ & $\begin{array}{l}\text { - Cobertura insuficiente da estratégia de saúde } \\
\text { GE, PA, USD. }\end{array}$ \\
\cline { 2 - 3 } & $\begin{array}{l}\text { Recursos humento dos serviços. Citado por } \\
\text { decitado por GE, PA, PSI; }\end{array}$ \\
\cline { 2 - 3 } & $\begin{array}{l}\text { Disponibilidade de } \\
\text { insumos }\end{array}$ & $\begin{array}{l}\text { - Oferta insuficiente de médicos e de outros } \\
\text { profissionais na atenção primária. Citado por } \\
\text { GE, PA, PSI, USD, PSII; }\end{array}$ \\
\cline { 2 - 3 } & $\begin{array}{l}\text { Acessibilidade } \\
\text { geográfica }\end{array}$ & $\begin{array}{l}\text { - Dificuldade de acesso geográfico, } \\
\text { distribuição inadequada no território. Citado } \\
\text { por GE, PSI, USD. }\end{array}$ \\
\hline
\end{tabular}




\begin{tabular}{|c|c|c|c|}
\hline to & Categorias & Subcategorias & Subcategorias emergentes \\
\hline & \multirow[t]{2}{*}{$\begin{array}{l}\text { Organizaçãoo } \\
\text { dos serviços }\end{array}$} & Visitas domiciliares & $\begin{array}{l}\text { - Quantitativo reduzido de visitas } \\
\text { domiciliares. Citado por USC, USD, PSI. }\end{array}$ \\
\hline & & Tempo de espera & $\begin{array}{l}\text { - Prolongado para a marcação de consultas. } \\
\text { Citado por USC, USD, PA; } \\
\text { - O horário de atendimento é insatisfatório. } \\
\text { Citado por USC, USD; } \\
\text { - Descumprimento do horário de trabalho dos } \\
\text { profissionais. GE, PA, PSI, USD, PSII, USC; }\end{array}$ \\
\hline & \multirow[t]{2}{*}{ População } & $\begin{array}{l}\text { Condição } \\
\text { socioeconômica }\end{array}$ & $\begin{array}{l}\text { - Condição socioeconômica precária. Citado } \\
\text { por PSI, USC. }\end{array}$ \\
\hline & & Informação & $\begin{array}{l}\text { - Faltam informaçóes sobre os serviços de } \\
\text { saúde. Citado por GE, PSI. }\end{array}$ \\
\hline
\end{tabular}

Gerentes (GE); Profissionais administrativos (PA), Profissionais de saúde Atenção Primária (PSI), Profissionais da Atenção Especializada (PSII), Usuários com cobertura (USC) e sem cobertura (USD).

Fonte: elaborado pelos autores.

\section{Elementos facilitadores de acesso aos}

\section{serviços de saúde da atenção primária}

A partir da análise das entrevistas, foram identificados alguns elementos que facilitam o acesso a APS relacionados à gestão da rede e aos serviços. Nos discursos, não emergiu elemento facilitador relacionado à população.

Quanto aos elementos facilitadores relacionados à gestão, uma importante subcategoria que emergiu dos discursos foi a própria existência da ESF. Apesar de terem expressado nos discursos a baixa cobertura da estratégia, sua implantação ampliou a oferta de serviços básicos, aproximou geograficamente o atendimento do usuário, diminuiu a fila nas unidades de saúde que já existiam e, principalmente, a busca dos hospitais.

Se não fosse esse posto eu acho que seria pior, porque se os hospitais já estão lotados com a quantidade de postos que tem nos bairros por ai, imagine se todos esses bairros não tivesse posto, todo mundo só poderia ir pro hospital. Então a situação seria mais feia, se não existisse esses postos em vários bairros como tem por aí. [...] com esses postos todos os hospitais estão cheios, imagine como seria. É muito critico. (USD 1).

Esse resultado mostra a importância da implementação da política no município, indo ao encontro dos resultados da pesquisa realizada por Almeida, 
Fausto e Giovanella (2011). Nela, profissionais e usuários também perceberam

a diminuição da procura por serviços especializados e hospitalares após a implantação da ESF, resultado que mostra que a ampliação da oferta da APS diminui a busca por outros serviços como primeiro contato.

No que se refere aos elementos facilitadores relacionados aos serviços, os principais que emergiram das entrevistas foram a oferta dos serviços disponíveis, a disponibilidade de insumos e a acessibilidade geográfica.

Com relação à oferta dos serviços disponíveis, apesar de ter emergido dos discursos como uma barreira de acesso, por ser um privilégio para alguns, os usuários não cobertos, principalmente, afirmaram que a comunicação informal com os funcionários possibilita o acesso a algum serviço de que tenham necessidade, o qual não conseguiriam pelas regras estabelecidas nas unidades.

Quando encontro uma pessoa amiga, assim como eu falei com você, que trato bem, aí eu falo com ela. Às vezes digo o que é que eu tenho: "Será que dá para você falar com a doutora para conseguir essa receita"? [...] A turma tem que fazer aquele "jogo de cintura”, se você não tiver aqueles seus conhecidos, aquela sua amizadezinha, eles não encaixam. (USD 6)

Essa afirmação também aparece em um estudo realizado por Pontes et al. (2009). Os usuários se referem ao alcance do atendimento intermediado pelo profissional de saúde e ao conhecimento como uma facilidade, o que pode ser caracterizado como privilégio, com a consequente associação do acesso a um sujeito individual (o profissional) e não à estrutura e à organização do sistema em si.

Quanto à acessibilidade geográfica, apesar de ter sido citada como uma barreira para alguns, os profissionais administrativos e os usuários, principalmente, expressaram que há facilidade de acesso, possibilitada pela presença das unidades básicas (tradicionais) e das USF dentro do território da população de referência, o que diminui a distância e facilita o deslocamento a pé, além de evitar a procura dos hospitais.

Não é longe para ele, porque a unidade só atende a população da redondeza. Então, para eles, aqui não é difícil acesso, porque eles moram na localidade, no bairro. Não atende fora, outras pessoas de fora, só a comunidade. (PA 3).

A proximidade física também foi citada em uma pesquisa realizada em quatro municípios brasileiros, na qual os usuários relataram chegar a pé às unidades (ALMEIDA; FAUSTO; GIOVANELLA, 2011). Escorel et al. (2007) também encontraram esse dado em uma pesquisa realizada em dez municípios no Brasil: 
mais de $90 \%$ das famílias entrevistadas afirmaram ser fácil chegar ao local e se deslocavam a pé até a unidade.

Além disso, em nosso estudo, os usuários destacaram que o trabalho realizado pelos ACS, presentes tanto na ESF como no programa de agentes comunitários de saúde, facilita o acesso aos serviços, por exemplo, através da marcação de consultas sem a necessidade de o usuário ir ao posto.

Essa agente de saúde, quando consegue marcar, ela vem trazer o papel em casa. [...] A gente vai pegar a ficha, vai marcar, aí ela telefona, fica tentando. Aí quando ela consegue, aí a agente de saúde vem e traz o papel, a ficha já marcada. (USC 2)

Tal resultado também aparece em Oliveira et al. (2012): o ACS é um agente importante de ligação entre a população e a USF. No entanto, Ferreira e Santo (2012) evidenciam que a prática do ACS é considerada um elemento facilitador principalmente por este facilitar a marcação de consultas. Portanto, a proposta de se superar o modelo médico-centrado e o papel do agente comunitário não vêm sendo significativos de maneira a serem valorizados pela população.

Tais resultados são apresentados no quadro-síntese abaixo (Quadro 2), com elementos facilitadores de acesso aos serviços de saúde no DS III do município de Recife, relacionados à gestão e à estrutura dos serviços.

QUADRO 2. Elementos facilitadores de acesso aos serviços de saúde relacionados com a gestão e com a estrutura dos serviços. Microrregião 3.3, DS III. Recife-PE, 2011

\begin{tabular}{|l|l|l|}
\hline Categorias & Subcategorias & Subcategorias emergentes \\
\hline \multirow{2}{*}{ Gestão } & Política de saúde & $\begin{array}{l}\text { - Existência da estratégia de saúde da família. } \\
\text { Citado por USD, PSI. }\end{array}$ \\
\hline \multirow{2}{*}{\begin{tabular}{l} 
Estrutura serviços \\
\cline { 2 - 3 }
\end{tabular}} & $\begin{array}{l}\text { Oferta dos serviços } \\
\text { disponíveis }\end{array}$ & $\begin{array}{l}\text { - A comunicação informal com os funcionários } \\
\text { da unidade. Citado por USD, USC. }\end{array}$ \\
\cline { 2 - 3 } & geográfica & $\begin{array}{l}\text { - A estratégia de saúde da família melhora o } \\
\text { acesso geográfico. Citado por PA, USC, USD; }\end{array}$ \\
\cline { 2 - 3 } & Recursos Humanos & $\begin{array}{l}\text { - A importância do papel dos ACS que estão } \\
\text { nas equipes de saúde da família (ESF) e no } \\
\text { programa de agente comunitário de saúde } \\
\text { (PACS). Citado por GE, PA, USC, USD. }\end{array}$ \\
\hline
\end{tabular}

Gerentes (GE); Profissionais administrativos (PA), Profissionais de saúde Atenção Primária (PSI), Profissionais da Atenção Especializada (PSII), Usuários com cobertura (USC) e sem cobertura (USD).

Fonte: elaborado pelos autores. 


\section{Considerações finais}

Apesar de todo um arcabouço político e institucional e de uma legislação já existente para a concretização do acesso dos usuários aos serviços de saúde do SUS, neste estudo, foi possível confirmar que há muitas barreiras que precisam ser enfrentadas pelas instituições públicas para ofertar assistência a todos, respeitando os princípios do SUS de universalidade, equidade e integralidade.

Pelos resultados da pesquisa, conclui-se que, para a grande maioria dos entrevistados, é difícil o acesso aos serviços de saúde de atenção primária na rede do município de Recife. Muitas barreiras foram identificadas e muito poucos foram os elementos facilitadores citados nas entrevistas realizadas.

Observou-se que muitos entrevistados relatam o subfinanciamento dos serviços de saúde, que tem como consequência muitas das barreiras estruturais dos serviços citadas pelos entrevistados, como a cobertura insuficiente da ESF, a falta de insumos e de médicos e outros profissionais. Outras barreiras - no caso, o tempo de espera, considerado elevado, e a pequena quantidade de visitas domiciliares, analisados como elementos organizativos dos serviços são consequência de um elemento estrutural dos serviços: a disponibilidade de recursos humanos. Isso demonstra que muitos dos fatores se inter-relacionam e se retroalimentam nas redes de saúde.

No que diz respeito aos elementos facilitadores, um achado relevante dos discursos dos entrevistados foi a importância da ESF. Para os informantes, a existência e a expansão da política é imprescindível para a oferta de serviços primários. Ela possibilita a prática de outros elementos que facilitam o acesso à saúde e que emergiram dos discursos, como o trabalho realizado pelo ACS e as visitas domiciliares de outros profissionais. Contudo, a cobertura da ESF foi considerada insuficiente para atender à totalidade da população, o que acarreta muitas áreas sem acesso aos serviços de saúde básica ou com grande dificuldade para utilizá-los.

Portanto, é necessário um reforço por parte da gestão para a manutenção do modelo de atenção à saúde já existente no município. Entretanto, é fundamental que se discuta como está se reestruturando a organização desta rede, para que se concretize e fortaleça o modelo de APS através da ESF, com o intuito de facilitar a trajetória dos usuários em busca do cuidado na rede. Também é importante 
a escuta da população através dos mecanismos de controle social, para que as mudanças ocorram de acordo com as reais necessidades dos usuários.

Finalizando, a pesquisa identificou que a APS precisa ser priorizada pela gestão para que a política da ESF realmente seja estruturante e orientadora da rede integral de serviços de saúde. Essa situação pode ser alcançada através de um maior financiamento do sistema - para a ampliação das equipes de saúde da família no município e melhoria da infraestrutura das unidades, garantindo condições dignas de atendimento para os usuários -, com investimento na organização do processo de trabalho dos profissionais e na educação permanente para a qualificação destes, no sentido de favorecer as ações voltadas para a prevenção e promoção da saúde individual e coletiva.

\section{Agradecimentos}

Agradecemos a todos que participaram do estudo Equity-LA, realizado em colaboração entre Consorci de Salud i Social de Catalunya (Espanha), o Instituto de Medicina Tropical de Amberes, Universidad del Rosario (Colômbia), Universidade de Pernambuco (Brasil), Centro de Pesquisa Aggeu Magalhães - Fiocruz e Instituto de Medicina Integral Prof. Fernando Figueira (Brasil), com o apoio das Secretarias de Saúde de Pernambuco e dos municípios. Agradecemos às pessoas entrevistadas, que aceitaram compartilhar com os investigadores suas opiniōes e conceitos, aos pesquisadores participantes, à Comissão Europeia, Programa FP7 e ao Ministério de Assuntos Exteriores de Espanha, cujo apoio financeiro foi imprescindível para a realização do estudo. A pesquisa que levou a estes resultados recebeu fundos do sétimo programa marco (FP7/2007-2013) contrato número 223.123².

\section{Referências}

ADAY, L. A.; ANDERSEN, R. A framework for the study of access to medical care. Health Services Research, Chicago, v. 9, n. 3, p. 208-220, 1974.

ALMEIDA, P. F. de; FAUSTO, M. C. R.; GIOVANELLA, L. Fortalecimento da atenção primária à saúde: estratégia para potencializar a coordenação dos cuidados. Revista Panamericana de Salud Publica, Washington, v. 92, n. 2, p. 84-95, 2011.

AZEVEDO, A. L. M.; COSTA, A. M. A estreita porta de entrada do Sistema Único de Saúde (SUS): uma avaliação do acesso na Estratégia de Saúde da Família. Interface: Comunicação, Saúde, Educação, Botucatu, v. 14, n. 35, p. 797-810, out./dez., 2010. 
BARDIN, L. Análise de Conteúdo. 1. ed. São Paulo: Edições 70, 2011.

BRASIL. Ministério da Saúde. Departamento de Atenção Básica. Histórico da cobertura da saúde da família. Disponível em: <http://dab.saude.gov.br/portaldab/historico_cobertura_ sf.php>. Acesso em: 9 out. 2013.

Portaria $n^{\circ}$ 4.279, de 30 de dezembro de 2010. Estabelece diretrizes para a organização da Rede de Atenção à Saúde no âmbito do SUS. Brasília, 2010. Disponível em: <http://cacphp.unioeste.br/pos/media/File/biociencias/Portaria_4279_rede_de_ atencao_a_saude.pdf>. Acesso em: 29 ago. 2012.

. Portaria $n^{\circ}$ 648, de 28 de março de 2006. Aprova a Política Nacional de Atenção Básica, estabelecendo a revisão de diretrizes e normas para a organização da Atenção Básica para o Programa Saúde da Família (PSF) e o Programa Agentes Comunitários de Saúde (PACS). Brasília; 2006. Disponível em: <http://dtr2001.saude.gov.br/sas/PORTARIAS/ Port2006/GM/GM-648.htm>. Acesso em: 18 out. 2013.

BRASIL. Secretaria de Gestão Estratégica e Participativa. Regulamentação da Lei 8.080 para fortalecimento do Sistema Único da Saúde: decreto 7508, de 2011. Revista Saúde Pública, São Paulo, v. 45, n. 6, p. 1206-1207, 2011 b.

COÊLHO, B. P.; COUTO, G. de A. O modelo de atenção e gestão em Recife: a dupla tarefa de produção da saúde e da produção dos sujeitos no Sistema Único de Saúde. Divulgação em Saúde para Debate, Rio de Janeiro, n. 44, p. 113-122, maio 2009.

COIMBRA, V. C. C. et al. Avaliação da estrutura - processo de acesso de usuários a uma unidade de saúde da família. Revista de pesquisa: cuidado é fundamental, v. 2, n.3, p. 10951107, jul.-set. 2010.

BRASIL. Ministério da Saúde. Conselho Nacional de Secretarias Municipais de Saúde. Comparação entre sistemas de serviços de saúde da Europa e o SUS: pontos para reflexão da agenda da atenção básica: Relatório de Oficina da Rede Américas, Belém: CONASEMS, 2008.

DRULLA, A. da G. et al. A visita domiciliar como ferramenta ao cuidado familiar. Cogitare Enfermagem, Curitiba, v. 14, n. 4, p. 667-74, out.-dez. 2009.

ESCOREL, S. et al. O Programa de Saúde da Família e a construção de um novo modelo para a atenção básica no Brasil. Revista Panamericana de Salud Publica, Washington, v. 21, n. 2, p. 164-176, 2007.

FERREIRA, J.; SANTO, W. E. Os percursos da cura: abordagem antropológica sobre os itinerários terapêuticos dos moradores do complexo de favelas de Manguinhos, Rio de Janeiro. Physis: Revista de Saúde Coletiva, Rio de Janeiro, v. 22, n. 1, p. 179-198, 2012.

GADELHA, C. A. G. et al.. Saúde e territorialização na perspectiva do desenvolvimento. Ciência \& Saúde Coletiva, Rio de Janeiro, v. 16, n.6, p. 3003-3016, 2011.

JESUS, W. L. A. de; ASSIS, M. M. A. Revisão sistemática sobre o conceito de acesso nos serviços de saúde: contribuições do planejamento. Ciência \& Saúde Coletiva, Rio de Janeiro, v. 15 , n. 1, p. 161-170, 2010. 
MOIMAZ, S. A. et al. Satisfação e percepção do usuário do SUS sobre o serviço público de saúde. Physis: Revista de Saúde Coletiva, Rio de Janeiro, v. 20, n. 4, p. 1419-1440, 2010. OLIVEIRA, L. S. et al. Acessibilidade a atenção básica em um distrito sanitário de Salvador. Ciência \& Saúde Coletiva, Rio de Janeiro, v. 17, n. 11, p. 3047-3056, 2012.

ORGANIZAÇÃO PANAMERICANA DA SAÚDE. Redes integradas de servicios de salud: conceptos, opciones de política y hoja de ruta para su implementación en las américas. Washington, DC: OPS, 2010, p. 35-54 (Serie Renovación de la atención primaria de la salud en las Américas, 4).

PINTO, E. S. G.; MENEZES, R. M. P. de; VILLA, T. C. S. Situação de trabalho dos profissionais da Estratégia Saúde da Família em Ceará-Mirim. Revista da Escola de Enfermagem da USP, São Paulo, v. 44, n. 3, p.657-64, 2010.

PONTES, A. P. M. et al. O princípio de universalidade do acesso aos serviços de saúde: o que pensam os usuários? Escola Anna Nery Revista de Enfermagem, Rio de Janeiro, v. 13, n. 3, p. 500-07, jul.-set. 2009.

SCHWARTZ, T. D. et al. Estratégia Saúde da Família: avaliando o acesso ao SUS a partir da percepção dos usuários da Unidade de Saúde de Resistência, na região de São Pedro, no município de Vitória (ES). Ciência \& Saúde Coletiva, Rio de Janeiro, v. 15, n. 4, p. $2145-$ $2154,2010$.

SHORTELL, S. M.; GILliES, R. R.; ANDERSON, D. A. The New World of Managed Care: creating organized delivery systems. Health Affairs, Millwood VA, v. 13, n. 5, p. 4664, 1994.

SILVA JÚNIOR, E. S. et al. Acessibilidade geográfica à atenção primária à saúde em distrito sanitário do município de Salvador, Bahia. Revista Brasileira de Saúde Materna Infantil, Recife, v. 10, supl.1, p. S49-S60, nov., 2010.

SILVA, S. F. Organização de redes regionalizadas e integradas de atenção à saúde: desafios do Sistema Único de Saúde (Brasil). Ciência \& Saúde Coletiva, Rio de Janeiro, v. 16, n. 6, p. 2753-2762, 2011.

TRAVASSOS, C.; OLIVEIRA, E. X. G. de; VIACAVA, F. Desigualdades geográficas e sociais no acesso aos serviços de saúde no Brasil: 1998 e 2003. Ciência \& Saúde Coletiva, Rio de Janeiro, v. 11, n. 4, p. 975-986, 2006.

VANDERLEI, L. C. de M.; NAVARETTE, M. L. V. Mortalidade infantil evitável e barreiras de acesso à atenção básica no Recife, Brasil. Revista de Saúde Pública, São Paulo, v. 47, n. 2, p. 379-389, 2013.

VÁZQUEZ, M. L. N. et al. Impact on equity of access and efficiency of Integrated Health care Networks (IHN) in Colombia and Brazil. Equity-LA project id: 223123 (2008-2013). 2013. Disponível em: <http://www2 equity-la eu/publications/technical-reports.> Acesso em: 6 fev. 2014. 
for analysis.Revista Panamericana de Salud Publica, Washington, DC, v. 26, n. 4, p. 360367, 2009.

VÁZQUEZ, M. L. N.; VARGAS, I. L. Redes integradas de servicios de salud: ¿Solución o problema? Ciencias de la Salud. Bogotá, v. 4, n. 1, p: 5-9, jan-jun. 2006.

\section{Nota}

${ }^{1}$ S. A. Vieira e M. R. Ferreira participaram da concepção do projeto e da análise e interpretação de dados, redação do artigo e revisão crítica relevante do conteúdo intelectual, bem como da aprovação final da versão a ser publicada. E. M. F. de Carvalho participou da redação do artigo, revisão crítica relevante do conteúdo intelectual e aprovação final da versão a ser publicada. E. A. P. Cesse participou da análise dos dados e da redação do artigo. E. S. V. de Brito e J.P. R. Braga participaram da coleta, análise e interpretação dos dados. 
Factors that influence access to primary care in the perspective of professionals and users of a health services network in Recife-PE, Brazil Despite all juridical-legal in the SUS apparatus that have been created to improve the population's access to health services, there are still many barriers that interfere in the concretion of the universal access to health care in Brazil. This study aims to analyze the factors which influence the access to primary care health services in Recife from the perspective of its professionals and users. This is a case study which used as data collection technique the semi structured interviews conducted with 46 informants. Bardin's content analysis was the method adopted to work with the data. The main barriers that emerged from the research were the underfunding of services, insufficient coverage family health strategy (FHS), and insufficient supply of professionals. Nevertheless, the most significant facilitating issues were: the existence of the FHS, the informal communication and the work of the community health agent. We conclude that even with the implementation of the FHS and the benefits generated, there is still the need for the strategy to be a management priority, with greater investments in the structure and organization of services offered to users in order for them to have universal access and equitable primary care services in the city of Recife.

> Key words: health services accessibility; Family Health Strategy; primary health care. 